\title{
Determining the Elastic Modulus and Hardness of an Ultrathin Film on a Substrate Using Nanoindentation
}

\section{Citation}

Li, Han, and Joost J. Vlassak. 2009. Determining the elastic modulus and hardness of an ultrathin film on a substrate using nanoindentation. Journal of Materials Research 24(3): 1114-1126.

\section{Published Version}

doi:10.1557/JMR.2009.0144

\section{Permanent link}

http://nrs.harvard.edu/urn-3:HUL.InstRepos:4262334

\section{Terms of Use}

This article was downloaded from Harvard University's DASH repository, and is made available under the terms and conditions applicable to Open Access Policy Articles, as set forth at http:// nrs.harvard.edu/urn-3:HUL.InstRepos:dash.current.terms-of-use\#OAP

\section{Share Your Story}

The Harvard community has made this article openly available.

Please share how this access benefits you. Submit a story.

\section{Accessibility}




\title{
Determining the elastic modulus and hardness of an ultra- thin film on a substrate using nanoindentation
}

\author{
Han Li , Joost J. Vlassak * \\ School of Engineering and Applied Sciences, Harvard University \\ Cambridge, Massachusetts, 02138, USA
}

\begin{abstract}
A data analysis procedure has been developed to estimate the contact area in an elasto-plastic indentation of a thin film bonded to a substrate. The procedure can be used to derive the elastic modulus and hardness of the film from the indentation load, displacement, and contact stiffness data at indentation depths that are a significant fraction of the film thickness. The analysis is based on Yu's elastic solution for the contact of a rigid conical punch on a layered half-space and uses an approach similar to the Oliver-Pharr method for bulk materials. The methodology is demonstrated for both compliant films on stiff substrates and the reverse combination and shows improved accuracy over previous methods.
\end{abstract}

Keywords: Nanoindentation, Elastic properties, Substrate effect, Thin films *Corresponding author. e-mail: vlassak@esag.harvard.edu 


\section{Introduction}

Since 1992, the analysis method proposed by Oliver and Pharr [1] has been established as the standard procedure for determining the hardness and elastic modulus from the indentation load-displacement curves for bulk materials. In the Oliver-Pharr method, the projected contact area between indenter tip and material is estimated using the equations for the elastic contact of an indenter of arbitrary shape on a uniform and isotropic half space [2]. The indentation modulus and hardness of the material can thus be calculated without the necessity of imaging the indentation after the experiment. The Oliver-Pharr method was initially developed for analyzing indentations in bulk materials, not for films on substrates, and no information about a possible substrate is included in the analysis. The Oliver-Pharr method is, however, frequently used by researchers to interpret indentations performed on thin films in an attempt to obtain approximate film properties regardless of the effect of substrate properties on the measurement. The accuracy of such a measurement depends on the film and substrate properties and on the indentation depth as a fraction of the total film thickness. In general, the error due to the substrate effect increases with increasing indentation depth and with increasing elastic mismatch between film and substrate [3-7]. To minimize the effect of the substrate on the measurement, the indentation depth is often limited to less than $10 \%$ of the film thickness [5]. This empirical rule is not always reliable, especially if the elastic mismatch between film and substrate is large. The $10 \%$ rule is also not useful for very thin films when experimental issues make it difficult to obtain accurate results for very shallow indentations. Evidently there exists a need for a method that can be used to analyze thin-film indentation data for indentation depths where the substrate effect cannot be ignored.

A number of studies with several different approaches to modeling the substrate effect have been reported [7-13]. King used numerical techniques to model the elastic indentation of a layered half space with flat-ended punches of various cross-sections [8]. The depth dependence of the effective indentation modulus of the composite system $M_{\text {eff }}$ was represented numerically as a function of the punch size $a$ normalized by the film thickness $t$ using the following phenomenological formula

$$
\frac{1}{M_{e f f}}=\frac{1-v_{f}^{2}}{E_{f}}\left(1-e^{-\psi \frac{t}{a}}\right)+\frac{1-v_{s}^{2}}{E_{s}} \cdot\left(e^{-\psi \frac{t}{a}}\right),
$$

where the subscripts $f$ and $s$ refer to the film and substrate, respectively. $E$ represents Young's modulus and $v$ Poisson's ratio. The contribution of the substrate to the effective modulus is through the exponential terms on the right hand side of Eq. (1). The empirical parameter $\psi$ is a function of $a / t$ only, and needs to be calculated 
numerically.

Gao et al. studied a similar elastic indentation problem using an approximate firstorder perturbation method [9]. The effective indentation modulus of the film/substrate system was determined in closed form as the weighted average of the indentation moduli of the film and the substrate. Gao's approximation becomes increasingly inaccurate as the elastic mismatch between the film and the substrate increases [11]. $\mathrm{Xu}$ and Pharr later showed that the accuracy of Gao's expression for the effective indentation modulus could be improved by a slight modification of the formula [13].

Yu et al. solved the elastic contact problem of an axisymmetric indenter - flat, conical or spherical in shape - on a layered half space by first reducing the mixed boundary value problem to a Fredholm integral equation of the second kind using the Papkovich-Neuber potentials, and then solving the integral equation numerically [10, 14]. Yu's solution can be regarded as a generalization of Sneddon's solution for a film/substrate composite and can be used to calculate the relations between indentation load, depth and contact stiffness for any combination of film and substrate. For the special case where the film has the same properties with the substrate, Yu's solution reduces to Sneddon's solution.

Saha and Nix adopted King's results in order to analyze elasto-plastic indentations performed with a Berkovich punch [7]. In their analysis, the film thickness in King's solution was replaced by the film thickness minus the instantaneous indentation depth. This is equivalent to assuming that the elastic recovery during unloading from an elasto-plastic indentation with a Berkovich indenter can be modeled by an elastic indentation with a flat-ended punch located at the tip of the Berkovich indenter. This assumption overestimates the substrate effect at relatively deep indentation depths [7, 12].

Chen and Vlassak [11] modeled the elasto-plastic indentation of a film on a substrate using finite elements. They demonstrated that the elastic unloading process in an elasto-plastic indentation was well approximated by Yu's elastic contact solution, which provided a unique relationship between the contact stiffness and contact area even in the presence of significant pile-up. This relationship was later adopted by Han et al. [12] to determine the hardness of a thin film on an elastically mismatched substrate. Instead of using finite elements, Han et al. derived the relationship between contact stiffness and area from Yu's analysis. Using Han's method, the instantaneous projected contact area can be estimated from the contact stiffness. Han's method cannot, however, be used to measure the indentation modulus of the film a priori. Instead, the method requires that the elastic properties of the film and substrate be known beforehand. Moreover, the precise definition of film thickness to be used in 
Yu's solution for the analysis of an elasto-plastic indentation is not clear: the local film thickness is not uniform and keeps changing as the indentation proceeds as a result of plastic deformation.

In this paper, we present a new data analysis procedure based on Yu's elastic solution to derive the projected contact area in an elasto-plastic indentation and to extract the elastic modulus and hardness of a film on a substrate. The procedure is in concept very similar to the classical Oliver-Pharr analysis except that it is based on Yu's elastic solution rather than Sneddon's solution. The data analysis procedure differs from Han's method in that it requires no prior knowledge of the film stiffness. The $p$ aper is organized as follows. We begin with a brief review of the Oliver-Pharr method. This section is followed by a summary of Yu's analysis and some relevant exact results for the elastic indentation of a film on a substrate. We then present a procedure for analyzing elasto-plastic indentation data and discuss the basic assumptions inherent to the procedure. Finally, the effectiveness of the new procedure is demonstrated experimentally for both compliant films on stiff substrates and vice versa.

\section{Theory}

\subsection{A brief review of the Oliver-Pharr method}

In the Oliver-Pharr method, Sneddon's elastic solution for the indentation of an isotropic half space [2] is used to relate the contact stiffness $S$ and the projected contact area $A$ between indenter and half space to the indentation modulus $M$ of a homogeneous material:

$$
M=\frac{\sqrt{\pi}}{2} \cdot \frac{S}{\sqrt{A}},
$$

where the indenter tip is assumed to be rigid. Equation (2) is valid independent of th e precise shape of the indenter, as long as it is smooth and axisymmetric [15]. If the indenter is not

axisymmetric, a correction factor $\beta$ is needed in Eq. (2) [8, 16]. For an isotropic mat erial, the indentation modulus $M$, so defined, is equal to the plane-strain modulus $E /\left(1-v^{2}\right)$. If the tip is not rigid, a reduced modulus, $M_{r}$, should be used in Eq. (2) to compensate for the finite compliance of the tip [1], i.e.

$$
M_{r}=\frac{\sqrt{\pi}}{2} \cdot \frac{S}{\sqrt{A}}, \text { with } \quad M_{r}=\left(M^{-1}+M_{t i p}{ }^{-1}\right)^{-1}
$$

In an elasto-plastic indentation, $S$ is measured as the derivative of the indentation load $P$ with respect to the elastic displacement on unloading. Pharr later pointed out that the elastic unloading from a plastic impression is equivalent to the elastic 
indentation of a flat surface by an effective punch, the shape of which is determined by the plastic properties of the material [17]. Since Eq. (2) does not depend on the precise shape of the punch, it can indeed be used in conditions where the physical surface is perturbed by a hardness impression. To determine the projected contact area $A$, one needs to determine the contact depth, $h_{c}$, i.e., the depth over which the indenter makes contact with the material. The contact depth can be estimated as

$$
h_{c}=h-h_{s},
$$

where $h$ is the total indentation displacement, and $h_{s}$ is the elastic deflection of the surface, which can be calculated from the elastic contact problem using

$$
h_{s}=\varepsilon \frac{P}{S} \text {. }
$$

In Eq. (4), $P$ is the indentation load and $\varepsilon$ is a constant that depends only on the shape of the indenter; $\varepsilon=0.72$ for a conical tip and 0.75 for a paraboloid tip [1]. Once $h_{c}$ has been calculated, the projected contact area, $A$, can be determined from the shape of the indenter, i.e., $A=f\left(h_{c}\right)$, where $f$ is the area function of the indenter, which describes the cross-sectional area of the indenter as a function of the distance to the indenter tip. Once the contact area is known, the indentation modulus and hardness of the material are readily calculated.

\subsection{Yu's analysis: elastic indentation problem and solution}

As mentioned in the introduction, the elastic indentation of a layered half-space is mathematically a mixed boundary value problem that can be reduced to a Fredholm integral equation of the second kind [10]:

$$
H(\tau)-\frac{1}{\pi} \int_{0}^{1}[K(y+\tau)+K(y-\tau)] H(y) d y=F_{0}(\tau) .
$$

The solution of the contact problem is given in terms of a function $H(\tau)$ that can be regarded as a normalized map of the pressure distribution within the contact region. The kernel of the integral equation, $K(u)$, is given by $[10,12]$

$$
K(u)=\frac{a}{t} \int_{0}^{\infty}\left(1-\frac{A \cdot 2 w+B \cdot \cosh (2 w)+C \cdot \sinh (2 w)}{D+C \cdot \cosh (2 w)+B \cdot \sinh (2 w)-A \cdot 2 w^{2}}\right) \cos \left(u w \frac{a}{t}\right) d w
$$

where 


$$
\begin{aligned}
& A=\left(1-\frac{M_{f}\left(1-v_{f}\right)}{M_{s}\left(1-v_{s}\right)}\right)\left(4 \frac{M_{f}\left(1-v_{f}\right)}{M_{s}\left(1-v_{s}\right)} v_{s}-3 \frac{M_{f}\left(1-v_{f}\right)}{M_{s}\left(1-v_{s}\right)}-1\right), \\
& B=8 \frac{M_{f}\left(1-v_{f}\right)}{M_{s}\left(1-v_{s}\right)}\left(v_{f}-1\right)\left(v_{s}-1\right), \\
& C=3-4 v_{f}+\frac{M_{f}\left(1-v_{f}\right)}{M_{s}\left(1-v_{s}\right)}\left[2+3 \frac{M_{f}\left(1-v_{f}\right)}{M_{s}\left(1-v_{s}\right)}-4 v_{f}-4 v_{s}\left(1+\frac{M_{f}\left(1-v_{f}\right)}{M_{s}\left(1-v_{s}\right)}-2 v_{f}\right)\right], \\
& D=\left[\frac{M_{f}\left(1-v_{f}\right)}{M_{s}\left(1-v_{s}\right)}\right]^{2}\left(4 v_{s}-3\right)-2 \frac{M_{f}\left(1-v_{f}\right)}{M_{s}\left(1-v_{s}\right)}\left(2 v_{f}-1\right)\left(2 v_{s}-1\right)+4 v_{f}\left(2 v_{f}-3\right)+5 .
\end{aligned}
$$

In these expressions, $M_{f}$ and $M_{s}$ are the indentation moduli of the film and the substrate respectively; $v_{f}$ and $v_{s}$ are the respective Poisson's ratios. The right hand side of Eq. (5) is determined by the shape of the indenter. For a conical punch, for instance,

$$
F_{0}(\tau)=1-\gamma \tau
$$

while for a spherical punch of radius $R$,

$$
F_{0}(\tau)=1-\gamma \tau \cdot \frac{\ln (1+\tau \rho)-\ln (1-\tau \rho)}{\ln (1+\rho / \gamma)-\ln (1-\rho / \gamma)}
$$

In these equations, $\rho=a / R$ and $\gamma$ is the ratio of the contact radius for an indentation in a film on a substrate to the contact radius for an indentation of the same depth in a homogenous half space with the same properties as the film, i.e.,

$$
\gamma=a / a^{H} .
$$

The relationship between the contact radius $a^{H}$ and the indentation depth $h$ for an elastic indention in a homogenous half space depends on the indenter shape [18]. For a conical punch with half included angle $\theta$, one finds that

$$
h=\frac{\pi a^{H}}{2 \tan (\theta)},
$$

while for a spherical punch of radius $R$

$$
h=\frac{a^{H}}{2}\left(\ln \frac{1+a^{H} / R}{1-a^{H} / R}\right) .
$$

The integral equation in Eq. (5) can be solved numerically in the form of a Chebyshev series using El-Gendi's method [10, 14]. For conical and spherical 
indenters, the boundary condition of vanishing pressure at the contact periphery is expressed as

$$
H(1)=0 \text {. }
$$

The value of $\gamma$ is iterated until the solution satisfies Eq. (11). The basic solution to the contact problem includes the function $H(\tau)$ and the corresponding value of $\gamma$. The general form of the indentation load $P$ required for an indenter to penetrate to a depth $h$ is then

$$
P=\frac{4 G_{f} a h}{\left(1-v_{f}\right)} \int_{0}^{1} H(\tau) d \tau .
$$

By using Eqs. (9), (10) and (12), $P$ can be determined as a function of $h$ for a given indenter shape. The contact stiffness is then the derivative of $P$ with respect to $h$.

\subsection{Some useful results from Yu's solution}

In an elastic indentation of a homogeneous half-space, the elastic deflection of the surface $h_{s}$ is related to the indentation load and the contact stiffness through Eq. (4). For a film on a substrate, $h_{s}$ also depends on the elastic mismatch between the film and the substrate, necessitating a dimensionless correction factor $\xi$ in the expression for the surface deflection

$$
h_{s}=\xi\left(a / t, M_{f} / M_{s}, v_{f}, v_{s}\right) \varepsilon \frac{P}{S} .
$$

The elastic deflection of the surface is also related to the contact radius through the indenter geometry. For a conical punch with half included angle $\theta$, one has

$$
h_{s}=h-h_{c}=h-a / \tan \theta \text {. }
$$

Using Eqs. (9) through (14), $\xi$ can be calculated as a function of $a / t$ and for different levels of elastic mismatch between film and substrate. Results for a conical indenter are shown in Fig. 1(a). When the film has the same elastic properties as the substrate, $\xi$ is obviously equal to one. As $a / t \rightarrow 0$, the curves also satisfy the condition $\xi \rightarrow 1$ independent of the elastic mismatch between film and substrate. This extreme case corresponds to an indentation in the homogeneous film material, for which $\xi$ should be equal to one. A similar trend is observed for very large contact areas, where the effect of the film is negligible and the indenter is effectively probing the substrate. It is further evident from Fig. 1(a) that Poisson's ratio of the film has a relatively minor effect on the value of $\xi$, especially so if the film is stiffer than the substrate. Figure 1(b) shows $\xi$ as a function of a/t for several conical indenters 
with different half included angles $\left(\theta=60^{\circ}, 70^{\circ}, 80^{\circ}\right)$ and for a spherical indenter $(R / t=30)$. Evidently there is a small difference between conical and spherical indenters, but for conical indenters $\xi$ does not depend on the apex angle of the indenter.

Analysis of nanoindentation data requires the relationship between the contact stiffness and the contact radius. We have calculated the contact stiffness $S$ as a function of the contact radius for a series of film/substrate combinations for a conical indenter with a half included angle of $70^{\circ}$. The results are shown on a logarithmic scale in Fig. 2(a). It is clear that for small contact radii, the contact stiffness changes linearly with contact radius conform to Sneddon's equation for homogeneous materials. As the contact radius grows, the effect of the substrate becomes evident and the slopes of the curves gradually deviate from unity: the slopes increase for stiff films on compliant substrates and decrease for compliant films on stiff substrates. At very large contact radii, all curves again approach Sneddon's equation as the effect of the film fades.

For a given contact area, the contact stiffness of an indenter on a homogeneous and isotropic half space is independent of the precise indenter shape as long as the indenter is axisymmetric and the profile of the punch can be approximated by a halfspace - i.e., the apex angle $\theta$ should be close to $180^{\circ}$ for a conical punch, or $a / R \ll 1$ for a spherical punch [15]. Consequently, the contact stiffness of an arbitrary axisymmetric indenter on an elastic half space is equal to that for a flat-ended indenter with radius equal to the contact radius. The same conclusion holds true for the indentation of a film on a substrate: In Fig. 2(b), we show the $S \sim a$ relationship calculated from Yu's solution for a given film-on-substrate assembly $\left(M_{f} / M_{s}=0.5, v_{f}=v_{s}=0.25\right)$ indented by several conical $\left(\theta=60^{\circ}, 70^{\circ}, 80^{\circ}\right)$ and spherical indenters $(R / t=10,30)$. All curves overlap perfectly confirming that for films on substrates the contact stiffness is also independent of the indenter shape, at least within the context of linear elastic contact mechanics. This observation is easily rationalized based on Hill's cumulative superposition argument [19] and is the foundation of the new analysis method. When analyzing the elastic unloading process after an elasto-plastic indentation in a thin film, the relationship between contact stiffness and contact radius can be calculated without knowledge of the precise shape of the indentation, i.e., without knowledge of the plastic properties of the film.

\subsection{Application of the elastic indentation solution to the analysis of elasto-plastic indentations: the effective film thickness}

The idea of using an elastic solution in the interpretation of elasto-plastic indentation data is based on the fact that the displacement during the unloading segment of an indentation is elastic even if the material has undergone significant 
plastic deformation on loading - at least in the absence of time-dependent deformation or a strong Bauschinger effect [1, 8, 17]. The entire unloading process can be modeled as the elastic contact between a flat surface and an effective indenter, the shape of which depends on the elastic and plastic properties of the indented material [17]. After the discussion in the previous section, it is clear that this approach is also valid for the elasto-plastic indentation of a film on a substrate, as long as the presence of the substrate is taken into account in the elastic analysis.

There is, however, one added complication: Film thickness is well defined in an elastic indentation, but not so in an elasto-plastic indention, where the film between the indenter and the substrate has been thinned as a result of plastic flow. In order to apply Yu's solution for the indentation of a film on a substrate it is necessary to define and use an effective film thickness, $t_{\text {eff }}$, that captures this local thinning effect. Generally, $t_{e f f}$ is a function of the elastic and plastic properties of both film and substrate. Dimensional analysis shows that for a given indenter shape

$$
\frac{t_{\text {eff }}}{t}=f\left(\frac{\sigma_{y}^{f}}{E_{f}}, \frac{\sigma_{y}^{s}}{E_{s}}, v_{f}, v_{s}, \frac{h}{t}\right),
$$

where $\sigma_{y}$ refers to the flow stress of the film or the substrate depending on the superscript. We simplify this equation as follows

$$
\frac{t_{e f f}}{t}=1-\eta\left(\frac{\sigma_{y}^{f}}{E_{f}}, \frac{\sigma_{y}^{s}}{E_{s}}, v_{f}, v_{s}\right) \frac{h}{t},
$$

in the spirit that the effective thickness is equal to the actual film thickness for zero indentation depth and decreases monotonously with increasing depth. The dimensionless function $\eta$ quantifies the local thinning of the film as a result of plastic deformation of the film. The function depends on the mechanical properties of film and substrate and on the precise indenter shape. In general, it is necessary to know $\eta$ in order to use Yu's solution in the analysis of elasto-plastic indentations in thin films. One option is to calculate $\eta$ numerically using a finite element model. We will demonstrate below that it is possible to experimentally determine $\eta$ for a given materials system by performing a nanoindentation measurement.

Given Yu's solution, it is now possible to calculate the instantaneous contact radius during an indentation in one of two ways. First, the contact radius can be calculated directly from the instantaneous contact stiffness using the $S \sim a$ relationship shown in Fig. 2(a), if values for $\eta$ and the indentation modulus of the film, $M_{f}$, are assumed. We refer to the value of $a$ calculated using this approach as the theoretical value of $a$. 
Second, the contact radius can also be determined using a procedure similar to the Oliver-Pharr method [1]. Specifically, it is assumed that the elastic deflection of the contact periphery is the same for an elasto-plastic indentation as it is for an elastic indentation. Replacing the film thickness in Eq. (13) with the effective thickness, one finds the following expression for the elastic deflection in an elasto-plastic indentation

$$
h_{s}=\xi\left(\frac{a}{(t-\eta \cdot h)}, \frac{M_{f}}{M_{s}}\right) \varepsilon \frac{P}{S},
$$

where the dependence on the Poisson's ratios of film and substrate is not written explicitly. Consequently the instantaneous contact radius $a$ needs to satisfy the following implicit equation with $a$ as the only unknown,

$$
a=\left(h-\xi\left(\frac{a}{(t-\eta \cdot h)}, \frac{M_{f}}{M_{s}}\right) \varepsilon \frac{P}{S}\right) \tan \vartheta .
$$

In a real experiment, the indenter is of course not a perfect cone and it may be necessary to determine the instantaneous contact radius from

$$
a=\sqrt{\frac{A}{\pi}}=\sqrt{\frac{f\left(h-h_{s}\right)}{\pi}},
$$

where $f$ is the known area function of the indenter. As can be seen in Fig. 1(b), the error in the correction factor $\xi$ as a result of the imperfect tip shape is expected to be quite small. Equations (18) or (19) can be solved numerically if $\eta$ and the indentation modulus of the film are known (or assumed), and it provides another measure for the instantaneous contact radius. We refer to this value of the contact radius as the experimental value of $a$.

If the contact stiffness is known at each point of the indentation loading curve e.g., from a continuous stiffness measurement - then the two measures of the $a$ can be calculated at every point of the indentation loading curve resulting in two continuous $S \sim a$ curves. If the assumed values of $\eta$ and the indentation modulus $M_{f}$ are correct, both curves overlap. In actual practice, $M_{f}$ and $\eta$ can be treated as free parameters that need to be varied to achieve the best possible overlap between the two $S \sim a$ curves, i.e., to minimize the mean square error between the two curves. Once $M_{f}$ and $\eta$ have been determined, the contact radius $a$ can be calculated and the hardness is found as $H=P / \pi a^{2}$. A detailed step-by-step outline to implement all these procedures is included in the appendix; a software package that performs the necessary calculations is available at the archival web site www.iMechanica.org [20]. The method described here reduces to the standard Oliver-Pharr method [1] if the 
film has the same properties as the substrate. In this special case, Eq. (13) reduces to Eq. (4) and the contact stiffness is linearly proportional with the contact radius through Eq. (2).

\section{Experimental}

\subsection{Materials}

The method described in the previous section was used to analyze indentations in a number of thin films. Table 1 summarizes the various materials that were used for this purpose; in all cases, the substrate was (100) silicon. The indentation modulus of the silicon substrate was determined from a nanoindentation experiment using the Oliver-Pharr method. The $\mathrm{SiO}_{2}$ film was a $300 \mathrm{~nm}$ thermally grown oxide purchased from Silicon Quest International. The silicon nitride film was a stoichiometric amorphous nitride deposited onto a silicon substrate using an industrial low-pressure chemical vapor deposition process. The porous organosilicate glass (OSG) coatings were deposited using an industrial plasma-enhanced chemical vapor deposition (PECVD) process with diethoxymethylsilane as a precursor along with a proprietary aromatic-organic porogen precursor. By varying the porogen loading during the film deposition process, OSG coatings with two different levels of porosity were deposited: OSG-1 with a porosity of $27 \%$ and OSG-2 with a porosity of $20 \%$. OSG coatings with two different thicknesses were deposited. The thicknesses of all films were measured after the deposition process with a Woollam V-VASE32 spectroscopic ellipsometry (made by J. A. Woollam Co., Inc.).

\subsection{Methods}

All nanoindentation tests were conducted in the continuous-stiffness-measurement (CSM) mode using a Nanoindenter XP system (MTS System Corporation, Oak Ridge, TN) equipped with a diamond Berkovich indenter tip. The indentation-loading scheme was similar for all tests: the measurements started with an exponentially increasing load until a specified peak displacement was reached. The load was held constant for 10 seconds and then decreased at a constant rate to $5 \%$ of the peak load. At this point, the load was held constant for 1 minute to allow for thermal drift correction, followed by the final unloading step. During the loading segment, the contact stiffness was measured continuously by imposing a small displacement oscillation at $40 \mathrm{~Hz}$ on the otherwise monotonously increasing displacement. The CSM measurement mode was ideal for the present analysis method as it supplied a near continuous $S \sim a$ curve. If the CSM mode is not available, the method can of course also be applied to indentations with multiple unloading cycles. At least five indentations were made for each coating, and the results presented here are the average of the group. The 
indentations in the thick OSG films were analyzed using the Oliver-Pharr method, while the indentations in all other films were analyzed using the method described in the previous section. The Poisson's ratios used in these analyses are summarized in Table 2. The dimensionless correction factor $\xi$ used in the analysis was that for a conical indenter.

Calibration tests for the area function of the indenter and for the load frame compliance were performed on a fused silica specimen to ensure measurement accuracy. A value of 1.034 was used for the correction factor $\beta$ in Sneddon's equation. A detection accuracy of the initial contact position of smaller than $1 \mathrm{~nm}$ was achieved by monitoring the stiffness signal as the tip came into contact with the sample surface [21]. To characterize the morphology of the indentations, postindentation atomic force microscopy (AFM) was carried out with an Asylum MFP-3D Stand-Alone AFM system in tapping mode. For all samples negligible pile-up was detected around the indentations, making them ideal test beds for the present analysis method. All nanoindentation results are summarized in Table 2.

The elastic modulus of the $\mathrm{Si}_{3} \mathrm{~N}_{4}$ film was also measured independently by means of the plane-strain bulge test [22]. A rectangular $\mathrm{Si}_{3} \mathrm{~N}_{4}$ membrane $(2.0 \pm 0.05 \mathrm{~mm} \mathrm{x}$ $10 \pm 0.05 \mathrm{~mm}$ ) was micro-fabricated using standard lithography and anisotropic etching techniques and deformed through application of a uniform pressure [22]. The deflection of the membrane was measured using a $\mathrm{He}-\mathrm{Ne}$ interferometer and the applied pressure using a manometer with a resolution of $0.1 \mathrm{kPa}$. The stress-strain curve of the $\mathrm{Si}_{3} \mathrm{~N}_{4}$ film was extracted from the load-deflection data using the method described in reference [22]. The plane-strain modulus of the $\mathrm{Si}_{3} \mathrm{~N}_{4}$ film was calculated from a linear least squares fit to the stress-strain curve.

\section{Results and Discussion}

\section{1 $\mathrm{Si}_{3} \mathrm{~N}_{4} / \mathrm{Si}$ and $\mathrm{SiO}_{2} / \mathrm{Si}$}

Figure 3 shows the indentation load-displacement curves for the $\mathrm{SiO}_{2}$ film, the $\mathrm{Si}_{3} \mathrm{~N}_{4}$ film, and the bare silicon substrate. The three indentation curves are very similar with only slight deviations as a result of the $\mathrm{SiO}_{2}$ and the $\mathrm{Si}_{3} \mathrm{~N}_{4}$ films; the indentations in the $\mathrm{SiO}_{2}$ and the $\mathrm{Si}_{3} \mathrm{~N}_{4}$ films penetrate well into the underlying silicon substrate, which seems to dominate the load-displacement data. To better differentiate the contributions of the films to the indentation curves and to avoid complications by indentation-induced fracture of the films and substrate, only indentation data for displacements less than two thirds of the film thickness are used in the analysis.

The experimental contact stiffness is depicted in Fig. 4 as a function of indentation depth for the $\mathrm{SiO}_{2}$ film, the $\mathrm{Si}_{3} \mathrm{~N}_{4}$ film, and the bare silicon substrate. As 
expected, the contact stiffness for the silicon substrate increases linearly with increasing indentation depth. The $\mathrm{SiO}_{2}$ and $\mathrm{Si}_{3} \mathrm{~N}_{4}$ curves have a constant slope at shallow depths where the films dominate the response, but the slopes start to change at intermediate depths, where the substrate becomes increasingly important. Whether the curves are concave or convex depends on the stiffness of the film relative to that of the substrate. Figure 5 shows the best fit of the theoretical $S \sim a$ relationship derived from Yu's solution to the experimental $S \sim a$ curve (markers) for both the $\mathrm{SiO}_{2}$ film and the $\mathrm{Si}_{3} \mathrm{~N}_{4}$ film. The inset shows the same data plotted as $S / 2 a$ as a function of $a / t$ to further highlight the goodness of fit. It is evident from both graphs that agreement between the experimental and theoretical curves is very good except for very small depths. The small discrepancy between both curves arises mainly because the initial contact between indenter and film is purely elastic. In this case, use of the total indentation depth $h$ in the expression for the effective film thickness is inappropriate. If the plastic depth is used in Eq. (16) instead of the total indentation depth, much better agreement is obtained. Using the plastic indentation depth indeed ensures that the method converges to the exact result for elastic indentations. Estimating the plastic indentation depth for each point on the loading curve is nontrivial, however, and involves further assumptions on the shape of the unloading curve. Furthermore, if the initial elastic contact is not included in the data analysis, virtually identical values for stiffness and hardness are obtained. Given the additional assumptions, the computational effort, and the very small benefit, it is not worthwhile to account for elastic contact in the definition of the effective depth.

The ratio of the indentation moduli for the $\mathrm{Si}_{3} \mathrm{~N}_{4}$ film obtained from the fit in Fig. 5 is $1.36 \pm 0.01$, yielding an indentation modulus of $242.5 \pm 0.9 \mathrm{GPa}$ for the $\mathrm{Si}_{3} \mathrm{~N}_{4}$ film. The corresponding value of $\eta$ is 0.55 . The pressure-deflection data for the $\mathrm{Si}_{3} \mathrm{~N}_{4}$ membrane obtained in the plane-strain bulge test are shown in Fig. 6. The inset is the corresponding stress-strain curve, the slope of which yields a plane-strain modulus of $257.2 \pm 1.5 \mathrm{GPa}$. For an isotropic material, the plane-strain modulus obtained in the bulge test is equivalent to the indentation modulus obtained in a nanoindentation experiment. Evidently, the bulge test result is in good agreement with the nanoindentation measurements on the same film. The result also agrees well with the value of $241 \pm 3 \mathrm{GPa}$ reported by Vlassak and Nix for a similar silicon nitride film [23].

The fit of the experimental $S$ - $a$ data in Fig. 5 yields an indentation modulus of $65.4 \pm 0.7 \mathrm{GPa}$ for the $\mathrm{SiO}_{2}$ film. The corresponding value of Young's modulus is 63.1 $\pm 0.7 \mathrm{GPa}$, assuming a Poisson's ratio of 0.19 for the $\mathrm{SiO}_{2}$ film $[24,25]$. Table 3 gives an overview of Young's moduli reported in the literature for $\mathrm{SiO}_{2}$ films that were thermally grown under various conditions. Most results are in the range of 
$63 \sim 72 \mathrm{GPa}$, in reasonable agreement with our nanoindentation results. The value of $\eta$ obtained in the analysis is 0.5 , close to the value for the $\mathrm{Si}_{3} \mathrm{~N}_{4}$ film. The parameter $\eta$ was introduced to quantify the local thinning of the film caused by plastic deformation. A value of approximately 0.5 suggests that in this case the effective film thickness is best approximated by the simple average of $t$ and $t-h$, rather than by $t$ as in Han's method [12] or $t-h$ as in Saha's method [7].

Figure 7 compares the indentation moduli of the $\mathrm{Si}_{3} \mathrm{~N}_{4}$ and $\mathrm{SiO}_{2}$ coatings obtained using the new method with the values obtained from the Oliver-Pharr method as a function of relative indentation size $(a / t)$, with the bulge test measurement, and with plane-strain moduli taken from the open literature (shaded regions) [1, 24-28]. The Oliver-Pharr results behave as expected: at very shallow depths, the indentation moduli are equal to the true indentation moduli of the films, but they change quickly with increasing indentation depth and approach the substrate indentation modulus. At small indentation depths $(a / t<0.2)$, the Oliver-Pharr moduli are noisy and suffer from a relatively large measurement uncertainty, precluding an unambiguous extrapolation to zero indentation depth. In contrast, the results obtained using the new analysis appear more robust because indentation data over a wide range of depths are used to determine the indentation moduli and because the effect of the substrate has been accounted for.

Figure 8 shows the hardness of the $\mathrm{SiO}_{2}$ film as a function of indentation depth, calculated using both the Oliver-Pharr analysis and the new method. Hardness results for bulk fused silica are also shown for comparison. At very shallow indentation depths, all results converge. Because of the finite tip radius, the indenter behaves more like a sphere than a sharp cone. As expected for a Hertzian contact, the hardness (i.e., the average contact pressure, as defined in this study) of the $\mathrm{SiO}_{2}$ sample starts at zero for zero depth and increases with increasing indentation depth. Eventually the curves level off and the hardness reaches a plateau value. The hardness of the $\mathrm{SiO}_{2}$ film as determined using the new method is in very good agreement with the hardness of fused silica, while the hardness of the $\mathrm{SiO}_{2}$ film as determined using the Oliver-Pharr method rises much faster. As the indenter tip approaches the $\mathrm{SiO}_{2} / \mathrm{Si}$ interface, the hardness of the $\mathrm{SiO}_{2}$ film as determined using the new analysis also starts to deviate from the fused silica hardness, although it remains smaller than the hardness determined using the Oliver-Pharr method. It is not clear at present whether this deviation is caused by the constraining effect of the silicon substrate on plastic flow in the coating [29] or because the analysis method loses accuracy when the indenter tip depth approaches the film/substrate interface.

Figure 9 shows the hardness of the $\mathrm{Si}_{3} \mathrm{~N}_{4}$ film calculated using the Oliver-Pharr 
analysis and the new method, along with the hardness results for the silicon substrate. Both $\mathrm{Si}_{3} \mathrm{~N}_{4}$ hardness curves look qualitatively similar, independent of which method was used to analyze the $\mathrm{Si}_{3} \mathrm{~N}_{4}$ results: They both rise from zero hardness for the elastic contact conditions at shallow depths and approach the hardness of the substrate at large depths. The details of the curves, however, are quite different. The maximum hardness obtained using the new analysis method is in good agreement with the hardness values of 21-23 GPa reported in the literature for similar LPCVD $\mathrm{Si}_{3} \mathrm{~N}_{4}$ films [23], while the hardness obtained using the Oliver-Pharr analysis is significantly lower. As the indenter approaches the interface, the hardness obtained from the new analysis drops off and reaches the silicon hardness much more quickly than the Oliver-Pharr hardness. This observation agrees with finite element simulations performed by Chen and Vlassak [11] for the indentation of a hard film on a soft substrate. These simulations indeed show that the hardness decreases rapidly as the indenter approaches the film/substrate interface because of extensive plastic deformation in the softer substrate

\subsection{OSG/Si}

Figure 10 shows the indentation load-displacement curves for the OSG-1 and OSG-2 films. Results are shown for two different film thicknesses. For either material, the response of the $2300 \mathrm{~nm}$ film is close to that of the $280 \mathrm{~nm}$ film as long as the indentation depth is small and the influence of the substrate negligible. At larger depths, the substrate effect becomes obvious and the thinner films require a larger load for the indenter to penetrate to a given depth than the thicker films. At a depth of approximately $200 \mathrm{~nm}$, kinks can be observed in the indentation curves for the thinner films. These kinks are associated with delamination of the OSG films from the substrate. Consequently only data obtained for depths smaller than $200 \mathrm{~nm}$ are used in the analysis. Figure 11 shows the best fit of the experimental $S \sim a$ data (hollow symbols) with the theoretical relation based on Yu's solution (solid curves) for the $280 \mathrm{~nm}$ OSG films. The corresponding indentation moduli are $4.45 \pm 0.19 \mathrm{GPa}(\eta=1.1)$ and $7.07 \pm 0.46 \mathrm{GPa}(\eta=1.2)$ for the OSG-1 and OSG-2 films, respectively. As illustrated in the inset, small deviations can be observed in the fit for very shallow depths when the results are plotted as $S / 2 a$ vers us $a / t$. We again attribute these deviations to the use of the total indentation depth in the definition of the effective film depth - they have little or no effect on the calculated film modulus.

For the $2300 \mathrm{~nm}$ OSG films, the indentation moduli calculated using the OliverPharr method are highly repeatable and remain nearly constant over a depth range of 50 to $200 \mathrm{~nm}$. Evidently, the substrate effect is negligible over this range, consistent 
with previous experimental and FEM studies [7, 11]. The average value of the indentation modulus in the plateau region is $4.50 \pm 0.20 \mathrm{GPa}$ for the OSG-1 film and $7.35 \pm 0.42 \mathrm{GPa}$ for the OSG-2 film, in good agreement with the results obtained for the thinner films over the same range of indentation depths using the new analysis.

Figure 11 also demonstrates the importance of using the effective film thickness in the analysis. The dashed curves are theoretical predictions based on Yu's solution using the same indentation moduli as for the solid curves, but assuming a constant thickness that is equal to the total film thickness (i.e., $\eta=0$ ). It is evident that the results are nearly independent of $\eta$ for shallow indentations - the plastic displacements are very small and the effect of the substrate is insignificant. This is no longer the case for larger indentations, however, and local plastic deformation has to be accounted for to obtain an accurate estimation of the contact radius. Indeed, if $\eta$ is set equal to zero and only the film modulus is allowed to vary, no good fit to the experimental data can be obtained.

The fact that $\eta>1$ for the OSG films stands in contrast with the values obtained for the $\mathrm{SiO}_{2}$ and $\mathrm{Si}_{3} \mathrm{~N}_{4}$ films $(\eta \sim 0.5)$. This result indicates that nanoindentation experiments on OSG films are much more sensitive to substrate effects than experiments on $\mathrm{SiO}_{2}$ or $\mathrm{Si}_{3} \mathrm{~N}_{4}$ films. We suggest here that this difference arises because of the porosity of the OSG coatings. The stress state under an indenter is highly hydrostatic and causes porous materials like OSG to densify during the indentation process. This densification leads to a local increase in the stiffness of the OSG. Chen and Vlassak [30] studied the effect of densification on nanoindentation for bulk porous materials. Using finite element simulations, they demonstrated that a porous material densifies in a small region below the indenter. Even though the stiffness of the material increased locally as a result of the densification, it was shown that the effect on the overall contact stiffness was not significant because the densified region was small compared to the overall volume of porous material contributing to the elastic displacement field. While this argument may be valid for porous solids in bulk form, it may be less so for the indentation of thin porous films on hard substrates. For the latter, the densified region constitutes a bigger fraction of the total film volume contributing to the elastic displacement field. Furthermore, a hard substrate may enhance densification underneath the indenter and thus lead to a larger region of increased stiffness. A detailed study of the interactions between the substrate effect and densification of porous coatings is beyond the scope of this study. Even so, it is evident from the measurements that the proposed analysis method gives accurate results when the coatings are porous: with reference to Table 2, the experimental values obtained for thin films using the new method are in very good agreement with the values obtained for the thick films using the Oliver-Pharr 
technique. This agreement also indicates that densification does not have a significant effect on the experimental indentation moduli. The values reported in this study are therefore representative of the as-deposited porous OSG coatings, not the densified material.

\subsection{A few additional considerations}

The extraction of the indentation modulus of the film relies on a fit of the experimental $S \sim a$ relationship with the theoretical result derived from Yu's solution using $M_{f}$ and $\eta$ as fitting parameters. One may wonder if two or more distinct combinations of $M_{f}$ and $\eta$ could give a comparable quality of fit? Indeed, for the spe cial case of a compliant film on a stiff substrate, one might expect a combination of a smaller $M_{f}$ and a larger $\eta$ (or vice versa) than the actual values to also give a reasonable fit to the experimental stiffness data. Figure 12 shows a contour plot of the sum of the fit residues squared $\left(=\chi^{2}\right)$ for the $\mathrm{SiO}_{2}$ film, where the fitting parameters have been varied over a wide range. The plot shows a clear minimum corresponding to the optimum combination of $M_{f}$ and $\eta$, even though the contour lines are somewhat elongated as anticipated on the basis of physical arguments. Evidently, the optimum fitting parameters are well defined and unique over the range of parameters shown in Fig. 12. Similar results are also found for the $\mathrm{Si}_{3} \mathrm{~N}_{4}$ and OSG coatings. We expect the analysis method to be robust for a wide range of coating and/or substrate properties.

One limitation of the current analysis method is that it requires knowledge of the Poisson's ratio of the film. Poisson's ratio of a thin film is difficult to measure and is hardly ever known with any accuracy. The extraction of the indentation modulus of the film is, however, fairly insensitive to the precise value of Poisson's ratio and a rough estimate is usually sufficient to perform the analysis. For example, Fig. 13 shows a graph of the indentation moduli of the $\mathrm{SiO}_{2}$ and $\mathrm{Si}_{3} \mathrm{~N}_{4}$ films as a function of the value of Poisson's ratio assumed in the analysis. Evidently, a rough estimate of Poisson's ratio is more than adequate to determine the indentation modulus.

It has been reported that the residual stress can affect the overall indentation response of a material and hence the measured hardness and modulus[31]. In our analysis, the effect of plasticity is modeled phenomenologically through use of the effective film thickness and the parameter $\eta$. We therefore expect that different levels of residual stress in a film may well lead to different values of $\eta$. The current analysis method, however, does not account for plastic pile-up around the indenter. Consequently, any changes in pile-up behavior may lead to systematic errors in the analysis, as is also the case with the standard Oliver-Pharr technique. 


\section{Conclusions}

A new method for the analysis of nanoindentation of thin films on substrates is presented. The method is similar to the Oliver-Pharr analysis method, but makes use of Yu's solution for the elastic contact of an indenter on a coated half space instead of Sneddon's solution for a homogeneous half space. This modification of the analysis procedure makes the new method applicable to thin films on substrates over a much larger range of indentation depths: Where the Oliver-Pharr analysis is applicable only in the limit of vanishing indentation depths, the new method is valid for indentation depths that are a significant fraction of the total film thickness. As such, the new method is ideal for the analysis of indentation experiments in very thin films where the minimum indentation depth is set by equipment limitations. The new method is demonstrated experimentally for both compliant films on stiff substrates and the reverse combination, yielding indentation moduli in good agreement with independent measurements and literature values.

\section{Acknowledgements}

This research was supported by the Semiconductor Research Corporation (Task ID: 1339.001). The silicon nitride and the OSG film stacks were provided by Texas Instruments Incorporated. The authors would like to thank $\mathrm{N}$. Ota for help with the nanoindentation measurements and TY Tsui for assistance with the OSG coatings. 


\section{References}

1. Oliver, W.C. and G.M. Pharr, An Improved Technique for Determining Hardness and Elastic-Modulus Using Load and Displacement Sensing Indentation Experiments. Journal of Materials Research, 1992. 7(6): p. 15641583.

2. Sneddon, I.N., The relation between load and penetration in the axisymmetric Boussinesq problem for a punch of arbitrary profile. International Journal of Engineering Science, 1965 3: p. 47.

3. Burnett, P.J. and D.S. Rickerby, The Mechanical Properties of Wear-resistent Coatings .1. Modeling of Hardness Behavior. Thin Solid Films, 1987. 148(1): p. 41-50.

4. Fabes, B.D., W.C. Oliver, R.A. McKee, and F.J. Walker, The Determination of Film Hardness from the Composite Response of Film and Substrate to Nanometer Scale Indentations Journal of Materials Research, 1992. 7(11): p. 3056-3064.

5. Pharr, G.M. and W.C. Oliver, Measurement of Thin-film Mechanical Properties Using Nanoindentation. Mrs Bulletin, 1992. 17(7): p. 28-33.

6. Mencik, J., D. Munz, E. Quandt, E.R. Weppelmann, and M.V. Swain, Determination of elastic modulus of thin layers using nanoindentation. Journal of Materials Research, 1997. 12(9): p. 2475-2484.

7. Saha, R. and W.D. Nix, Effects of the substrate on the determination of thin film mechanical properties by nanoindentation. Acta Materialia, 2002. 50(1): p. 23-38.

8. King, R.B., Elastic Analysis of Some Punch Problems for a Layered Medium. International Journal of Solids and Structures, 1987. 23(12): p. 1657-1664.

9. Gao, H.J., C.H. Chiu, and J. Lee, Elastic Contact versus Indentation Modeling of Multilayered Materials. International Journal of Solids and Structures, 1992. 29(20): p. 2471-2492.

10. Yu, H.Y., S.C. Sanday, and B.B. Rath, The Effect of Substrate on the Elastic Properties of Films Determined by the Indentation Test - Axisymmetrical Boussinesq Problem. Journal of the Mechanics and Physics of Solids, 1990. 38(6): p. 745-764.

11. Chen, X. and J.J. Vlassak, Numerical Study on the Measurement of Thin Film Mechanical Properties by Means of Nanoindentation. Journal of Materials Research, 2001. 16(10): p. 2974-2982.

12. Han, S.M., R. Saha, and W.D. Nix, Determining hardness of thin films in elastically mismatched film-on-substrate systems using nanoindentation. Acta Materialia, 2006. 54(6): p. 1571-1581. 
13. Xu, H.T. and G.M. Pharr, An Improved Relation for the Effective Elastic Compliance of a Film/substrate System during Indentation by a Flat Cylindrical Punch. Scripta Materialia, 2006. 55(4): p. 315-318.

14. Elgendi, S.E., Chebyshev Solution of Differential, Integral and IntegroDifferential Equations. Computer Journal, 1969. 12(3): p. 282-\&.

15. Pharr, G.M., W.C. Oliver, and F.R. Brotzen, On the Generality of the Relationship among Contact Stiffness, Contact Area, and Elastic Modulus during Indentation Journal of Materials Research, 1992. 7(3): p. 613-617.

16. Vlassak, J.J. and W.D. Nix, Measuring the Elastic Properties of Anisotropic Materials by Means of Indentation Experiments. Journal of the Mechanics and Physics of Solids, 1994. 42(8): p. 1223-1245.

17. Pharr, G.M. and A. Bolshakov, Understanding nanoindentation unloading curves. Journal of Materials Research, 2002. 17(10): p. 2660-2671.

18. Harding, J.W. and I.N. Sneddon, The Elastic Stresses Produced by the Indentation of the Plane Surface of a Semi-infinite Elastic Solid by a Rigid Punch Proceedings of the Cambridge Philosophical Society, 1945. 41(1): p. 1626.

19. Hill, R. and B. Storåkers. A Concise Treatment of Axisymmetric Indentation in Elasticity. in Elasticity: mathematical methods and applications. 1990.Ian N.

Sneddon 70th birthday volume. G. Eason and R.W. Ogden. p 199-209.

20. url: http://imechanica.org/node/4050. "iMechanica" is a website hosted at Harvard School of Engineering and Applied Sciences dedicated to enhance communication and discussion among mechanicians

21. Oliver, W.C. and G.M. Pharr, Measurement of hardness and elastic modulus by instrumented indentation: Advances in understanding and refinements to methodology. Journal of Materials Research, 2004. 19(1): p. 3-20.

22. Xiang, Y., X. Chen, and J.J. Vlassak, Plane-strain bulge test for thin films. Journal of Materials Research, 2005. 20(9): p. 2360-2370.

23. Vlassak, J.J. and W.D. Nix, A New Bulge Test Technique for the Determination of Young Modulus and Poisson Ratio of Thin-Films. Journal of Materials Research, 1992. 7(12): p. 3242-3249.

24. Jaccodin.Rj and W.A. Schlegel, Measurement of Strains at Si-SiO2 Interface. Journal of Applied Physics, 1966. 37(6): p. 2429-\&.

25. Carlotti, G., L. Doucet, and M. Dupeux, Comparative study of the elastic properties of silicate glass films grown by plasma enhanced chemical vapor. Journal of Vacuum Science \& Technology B, 1996. 14(6): p. 3460-3464.

26. Petersen, K.E. and C.R. Guarnieri, Young's Modulus Measurement of Thin-films Using Micromechanics. Journal of Applied Physics, 1979. 50(11): p. 6761-6766. 
27. Petersen, K.E., Dynamic Micromechanics on Silicon-techniques and Devices. Ieee Transactions on Electron Devices, 1978. 25(10): p. 1241-1250.

28. Blech, I. and U. Cohen, Effects of Humidity on Stress in Thin Silicon Dioxide Films. Journal of Applied Physics, 1982. 53(6): p. 4202-4207.

29. Saha, R., Z.Y. Xue, Y. Huang, and W.D. Nix, Indentation of a Soft Metal Film on a Hard Substrate: Strain Gradient Hardening Effects. Journal of the Mechanics and Physics of Solids, 2001. 49(9): p. 1997-2014.

30. Chen, X., Y. Xiang, and J.J. Vlassak, Novel technique for measuring the mechanical properties of porous materials by nanoindentation. Journal of Materials Research, 2006. 21(3): p. 715-724.

31. Tsui, T.Y., W.C. Oliver, and G.M. Pharr, Influences of stress on the measurement of mechanical properties using nanoindentation .1. Experimental studies in an aluminum alloy. Journal of Materials Research, 1996. 11(3): p. 752-759. 


\section{Figures}

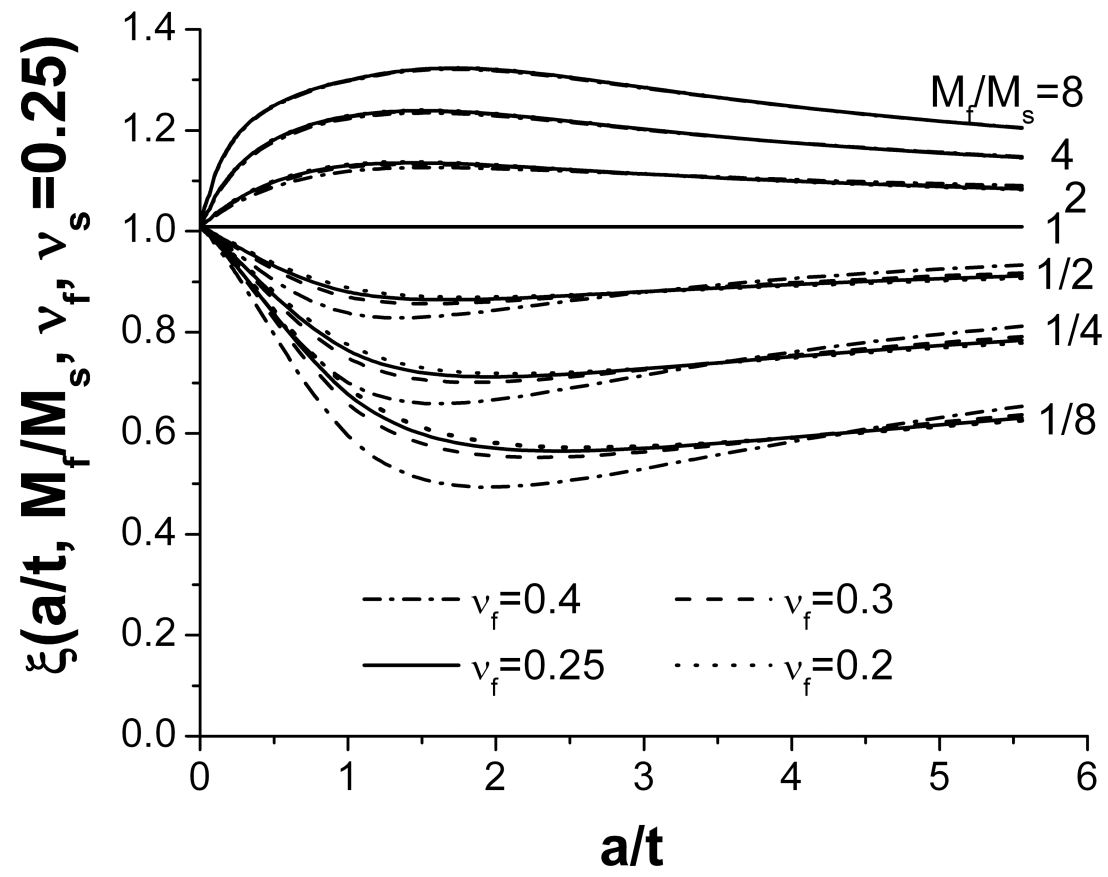

(a)

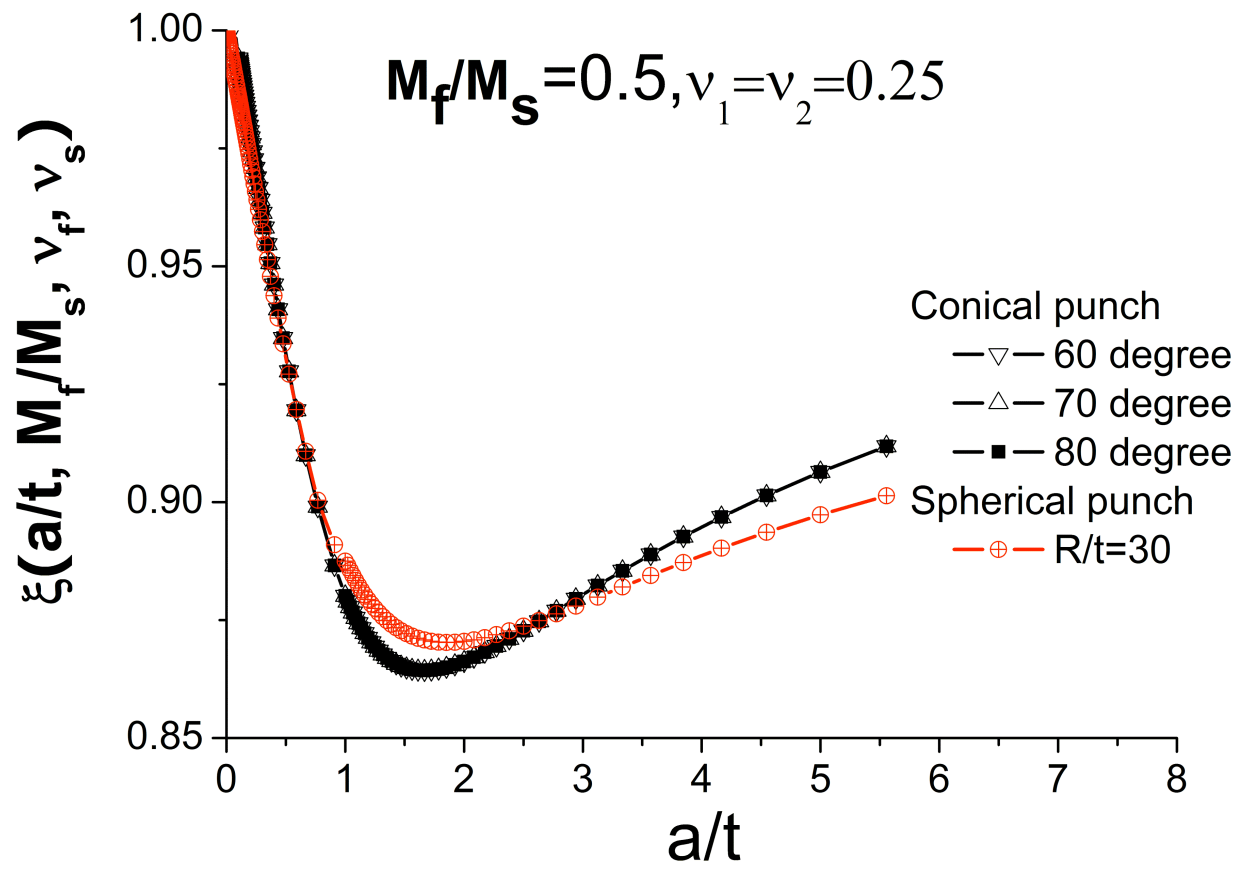

(b)

Fig. 1. The dimensionless correction factor $\xi$ for an elastic indentation as a function of normalized contact radius for (a) different elastic mismatch and a conical indenter, and for (b) various indenter shapes. 


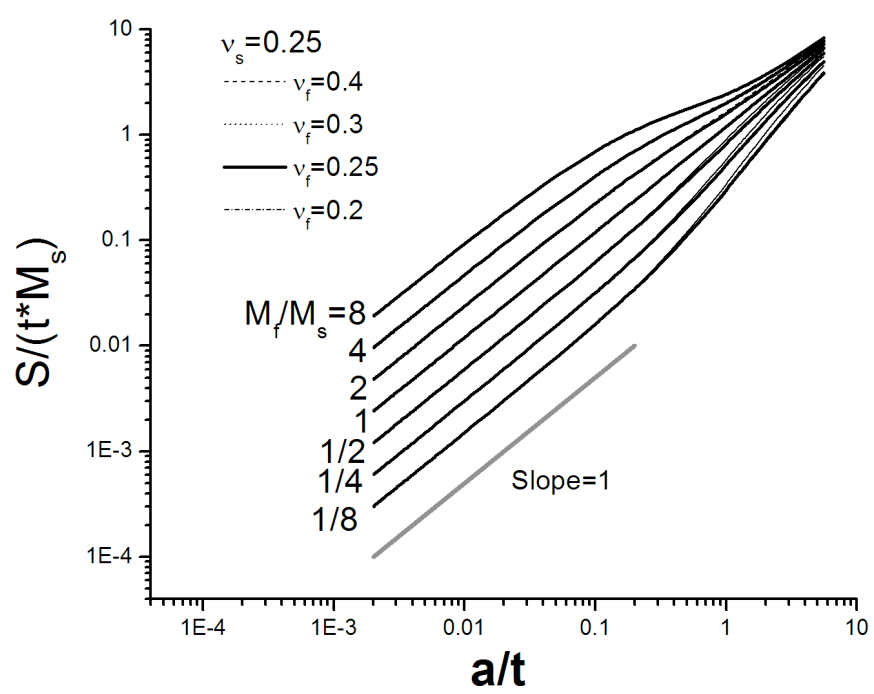

(a)

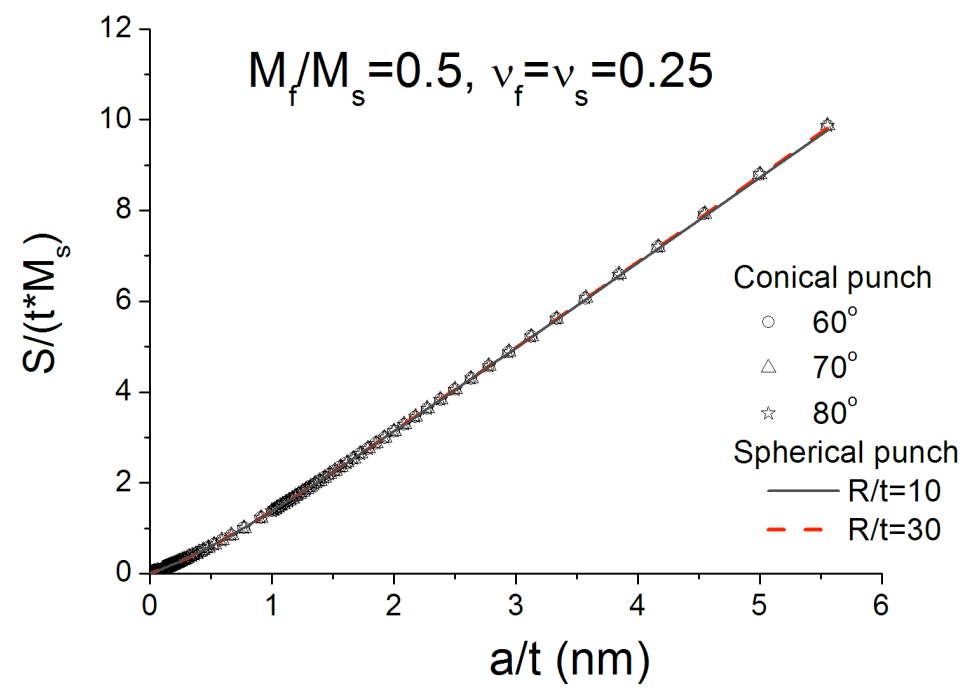

b)

Fig. 2. Normalized contact stiffness versus contact radius calculated from Yu's solution for (a) different elastic mismatches, and (b) various conical and spherical punches. 


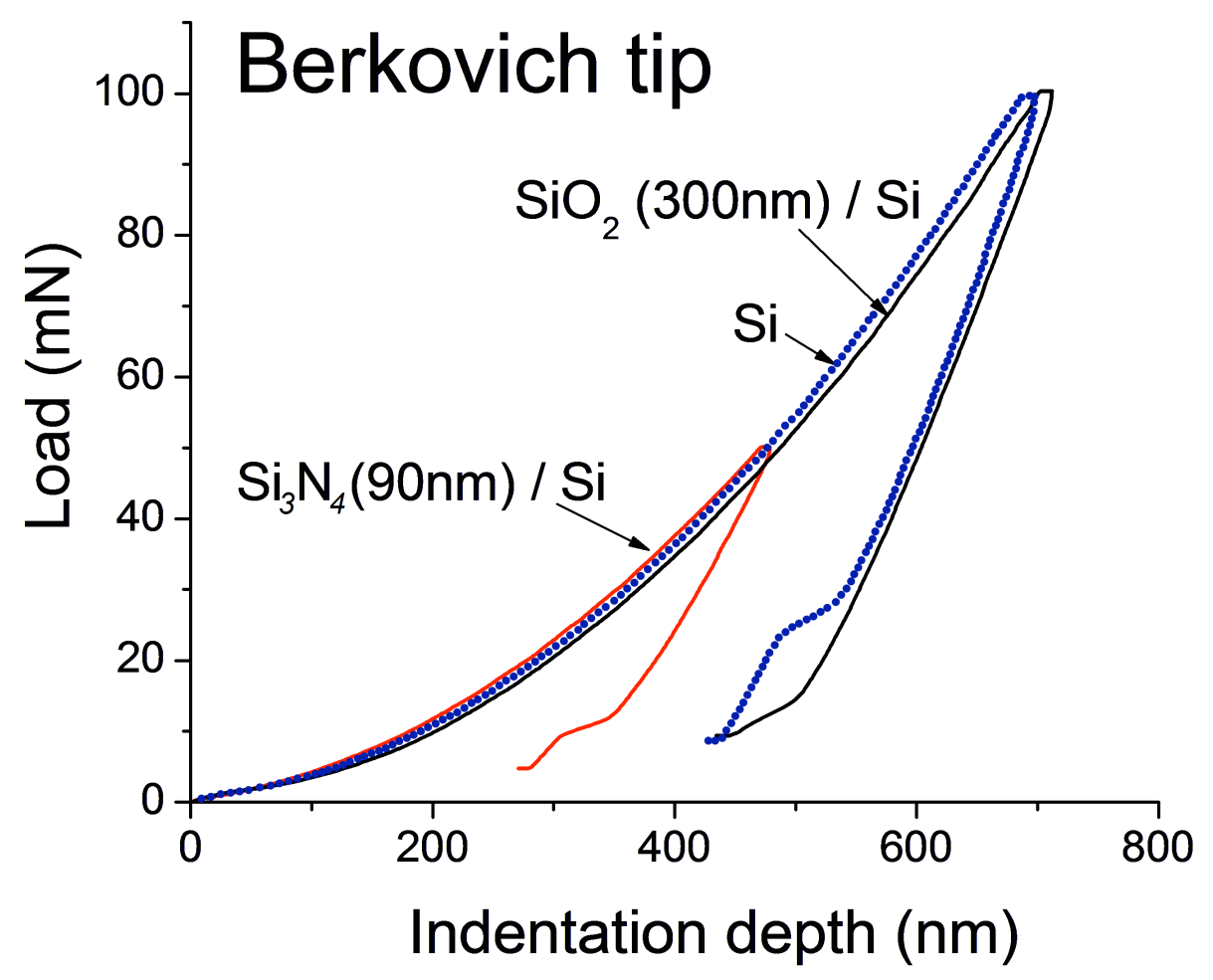

Fig. 3. Experimental load-displacement curves for the $\mathrm{Si}_{3} \mathrm{~N}_{4}$ and $\mathrm{SiO}_{2}$ films, and for the silicon substrate. 


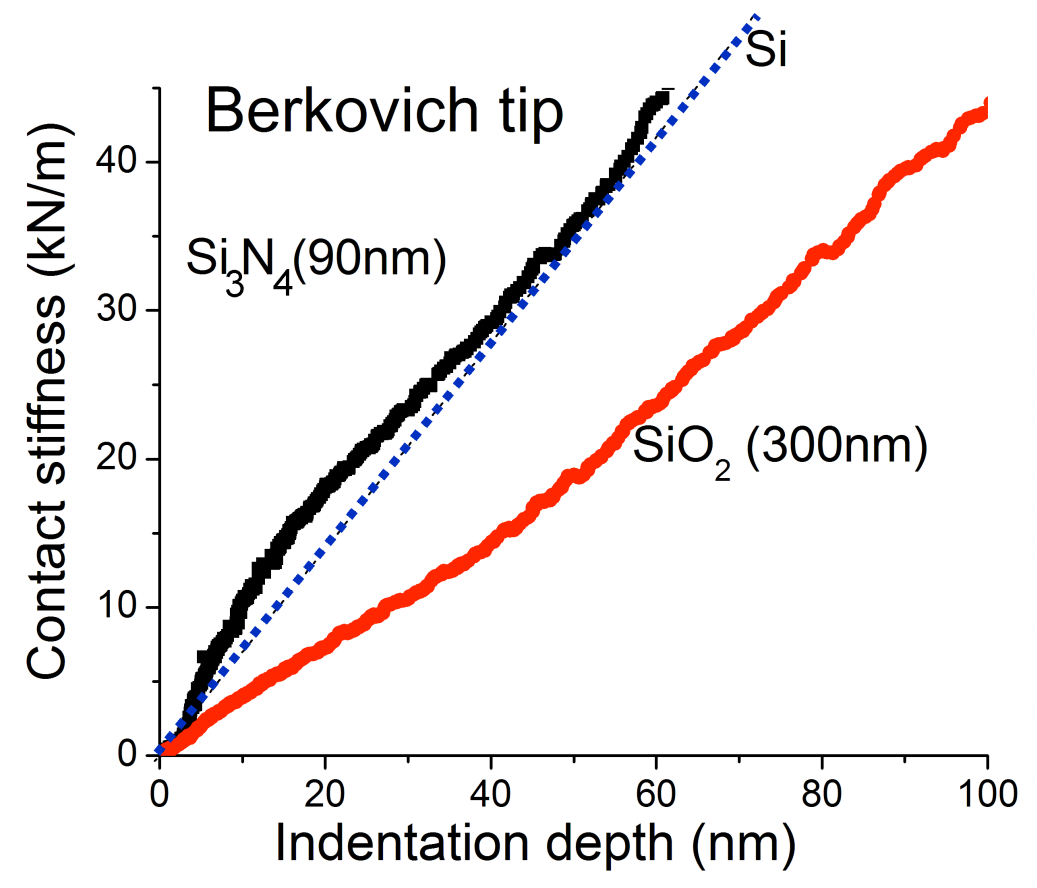

Fig. 4. Curves of the experimental contact stiffness versus indentation depth for the $\mathrm{Si}_{3} \mathrm{~N}_{4}$ film, the $\mathrm{SiO}_{2}$ film, and for the silicon substrate. 


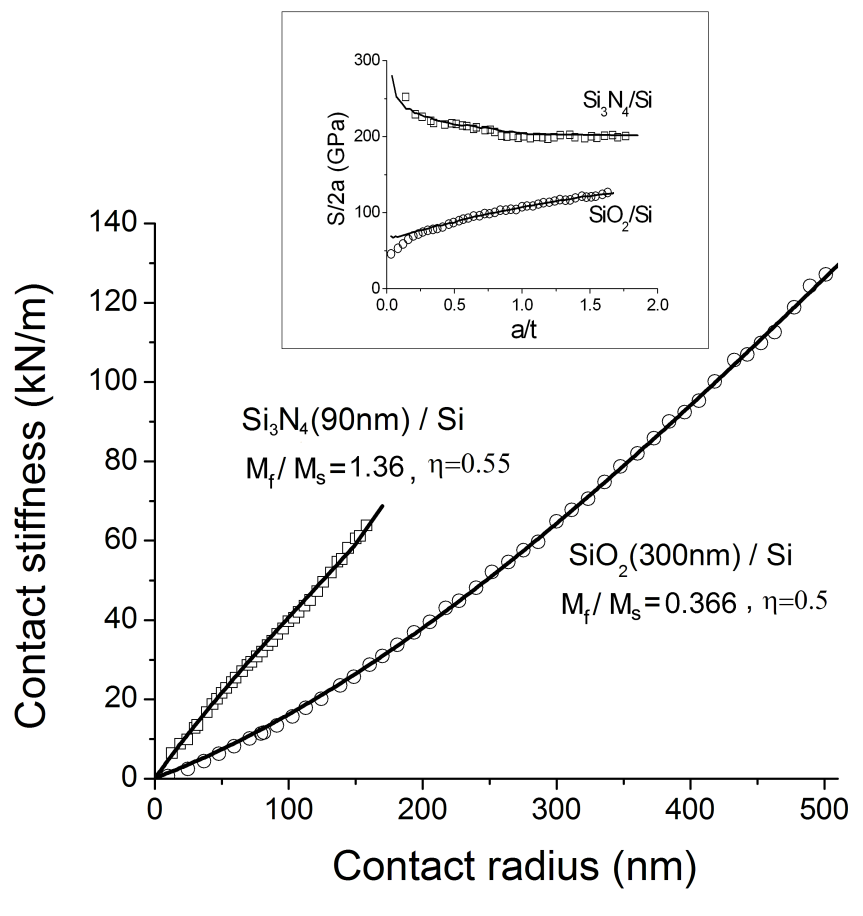

Fig. 5. Experimental (markers) and theoretical (solid curves) contact stiffness versus contact radius for the $\mathrm{Si}_{3} \mathrm{~N}_{4}$ and $\mathrm{SiO}_{2}$ samples. The inset presents the same data in the form of $S / 2 a$ versus $a / t$. 


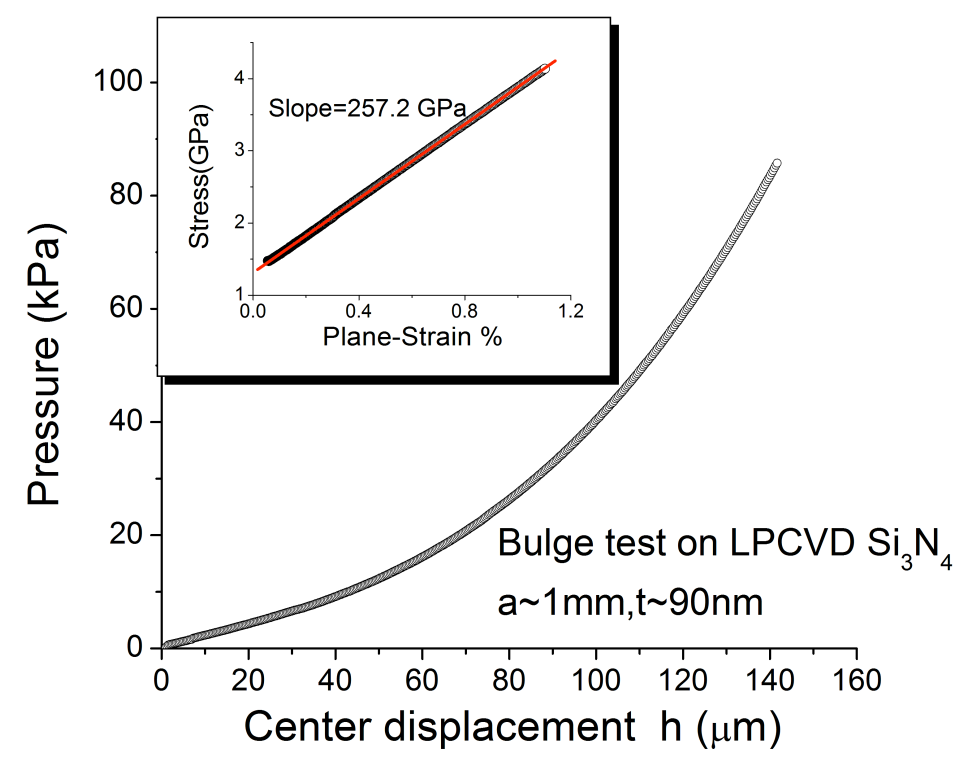

Fig. 6. The pressure-displacement curve for a freestanding LPCVD silicon nitride film obtained in the bulge test. The inset is the corresponding plane-strain stress-strain curve, yielding a plane-strain modulus of $257.2 \pm 1.5 \mathrm{GPa}$. 


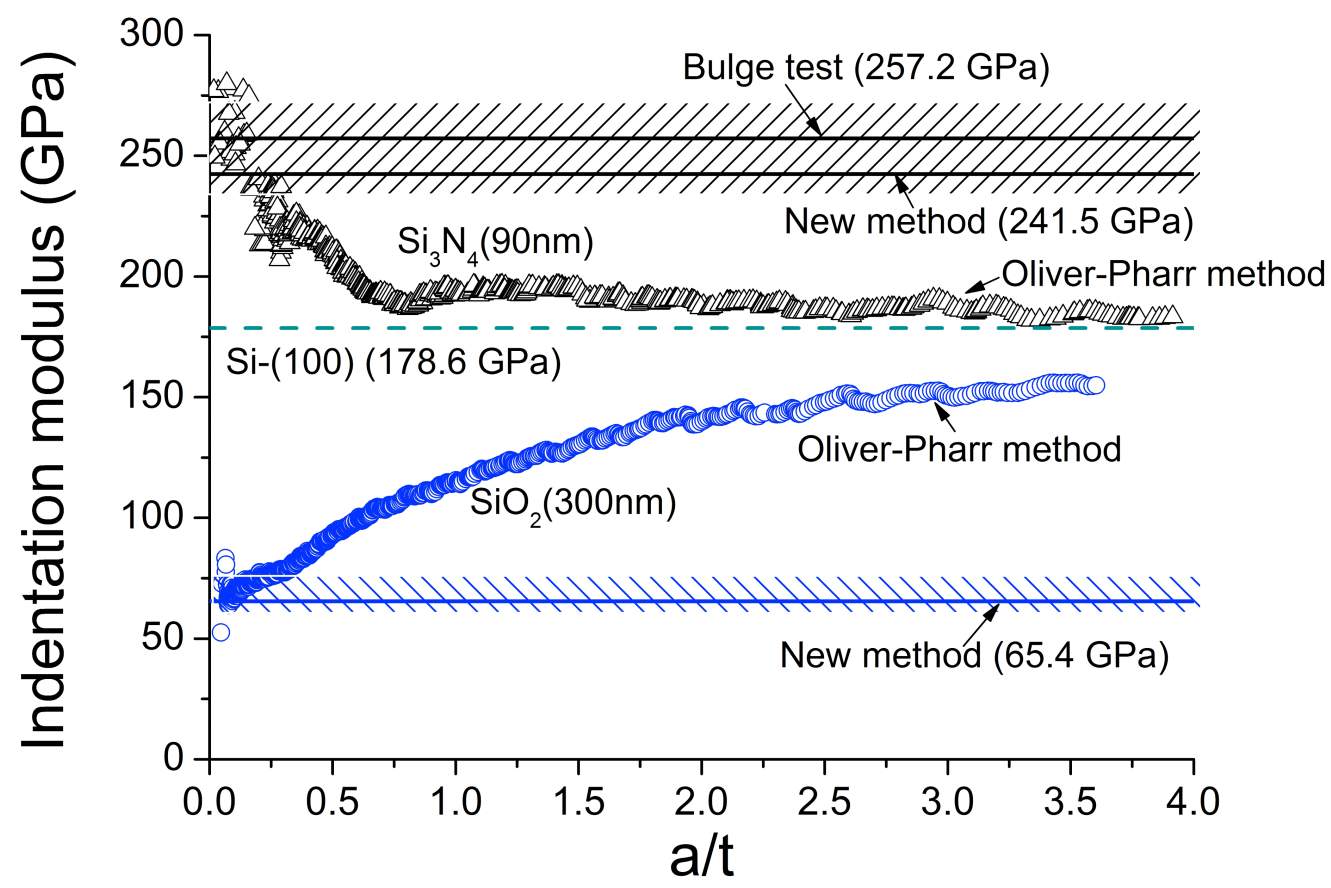

Fig. 7. The indentation modulus obtained with the Oliver-Pharr method as a function of contact radius normalized by film thickness, compared with the results obtained using the new method. The shaded regions represent the ranges of the $\mathrm{SiO}_{2}$ and $\mathrm{Si}_{3} \mathrm{~N}_{4}$ indentation moduli reported in the literature. 


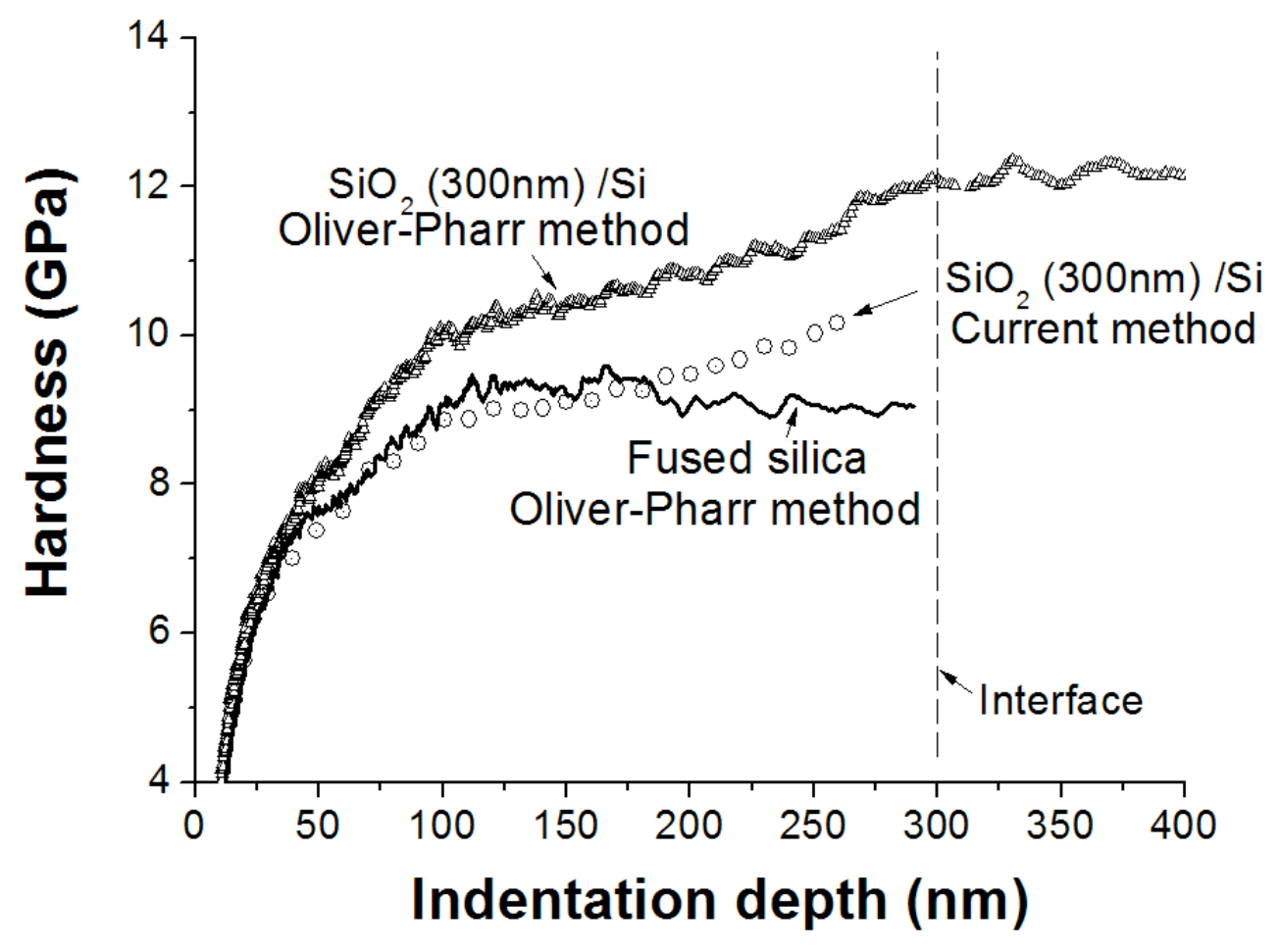

Fig. 8. The hardness of the $\mathrm{SiO}_{2}$ film as a function of indentation depth calculated using several methods. The hardness of bulk fused quartz is included for comparison.

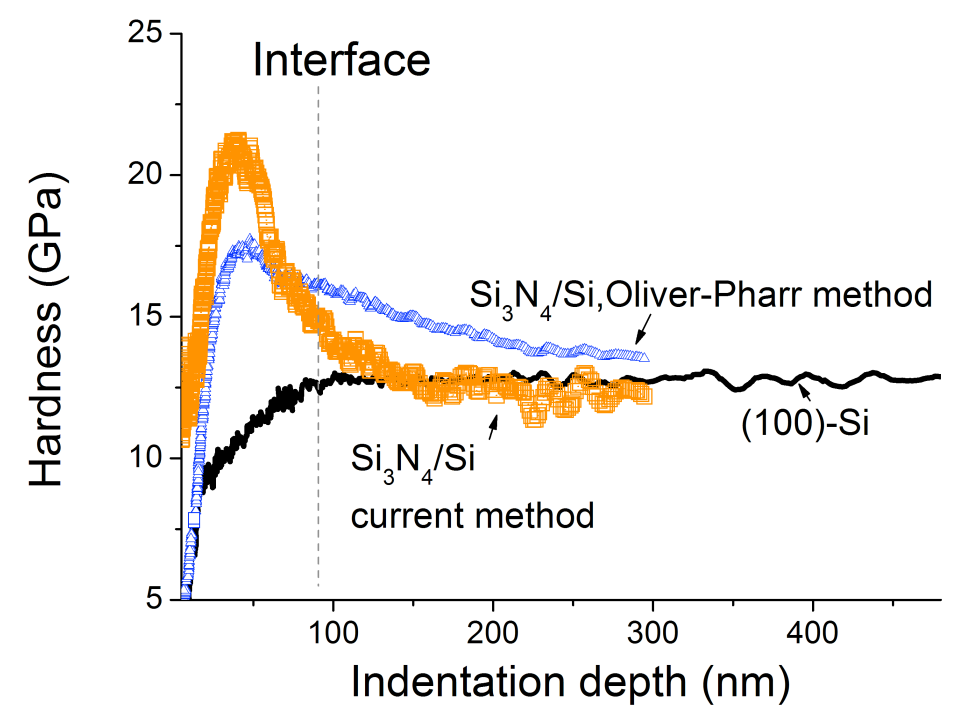

Fig. 9. The hardness of the $\mathrm{Si}_{3} \mathrm{~N}_{4}$ film as a function of indentation depth calculated using several methods. The hardness for the silicon substrate is included for comparison. 


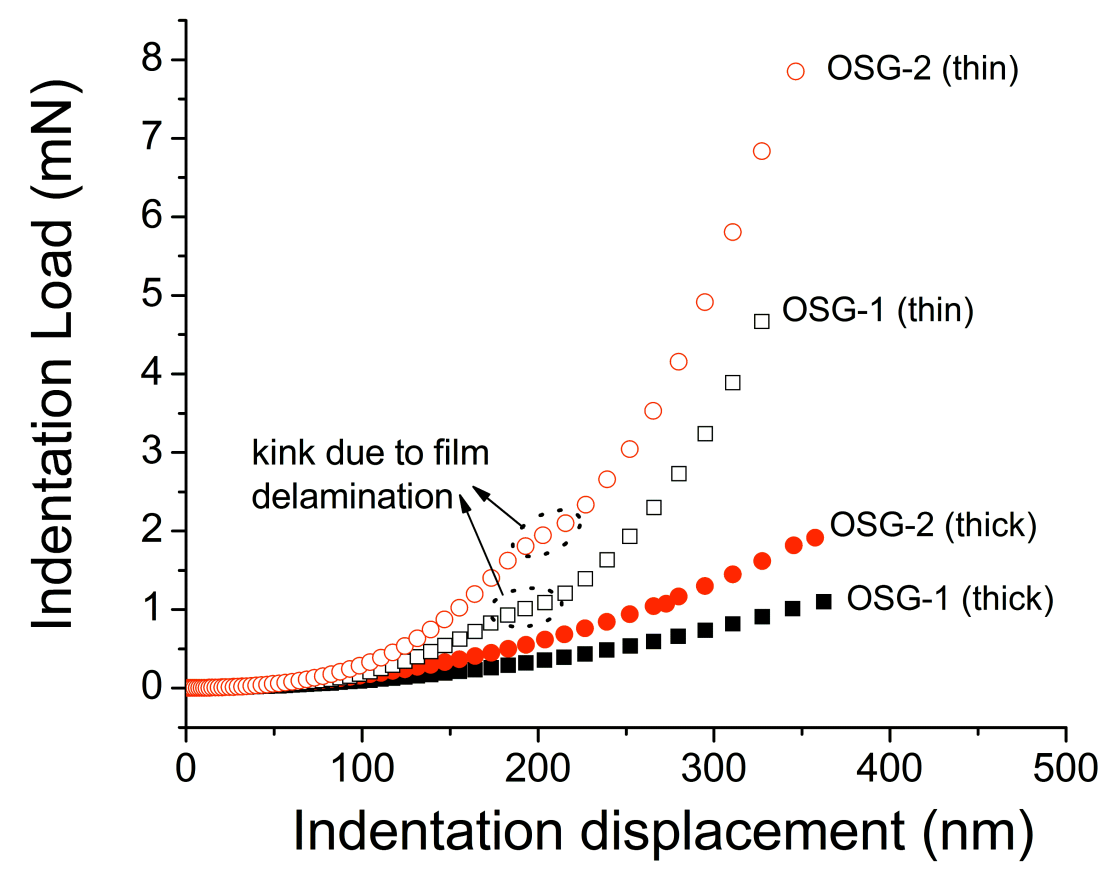

Fig. 10. Load-displacement curves for the two OSG films of the same properties but different thicknesses on silicon substrate, interfacial delamination at position circled. 


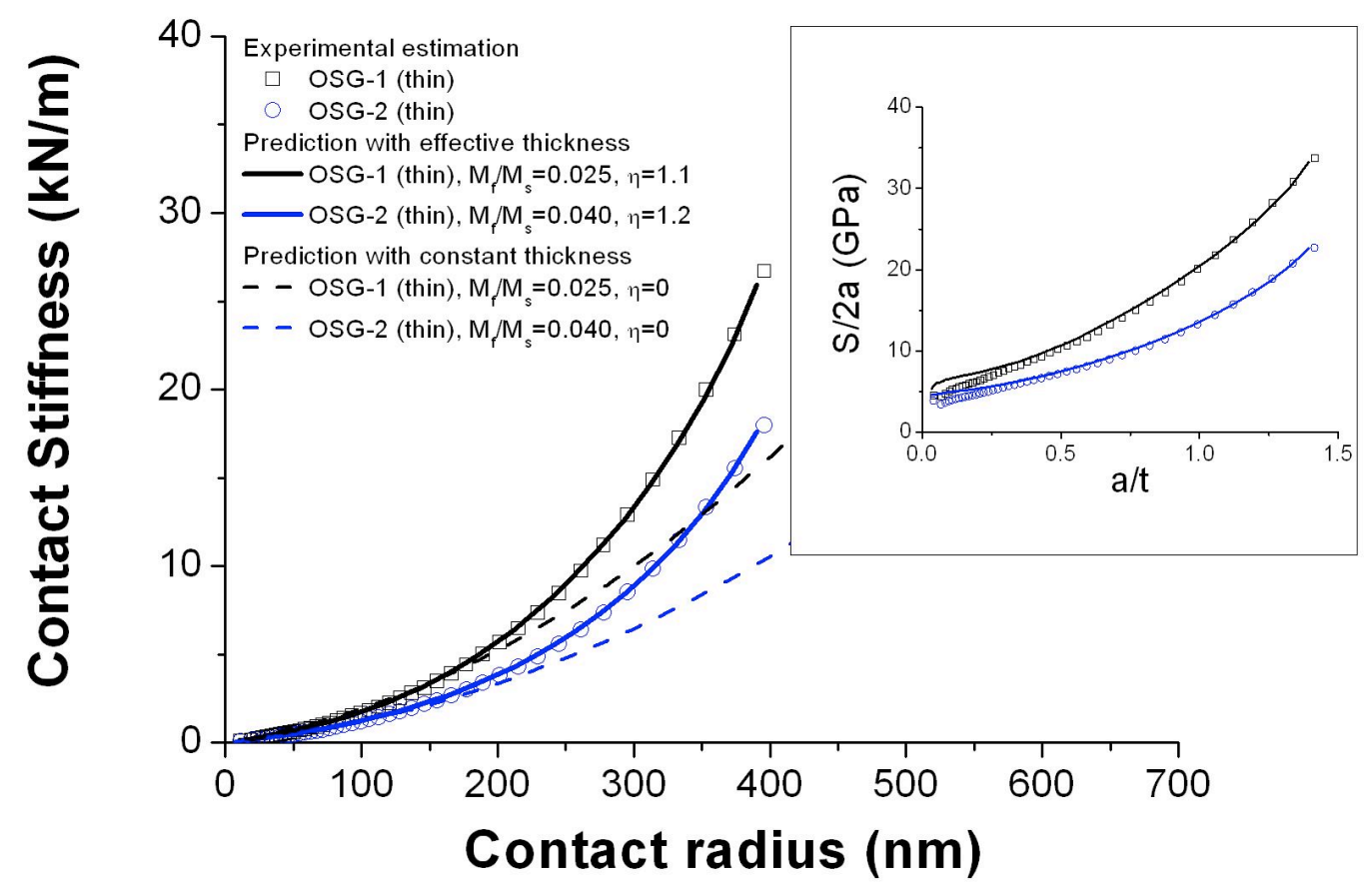

Fig. 11. Experimental and theoretical contact stiffness as a function of contact radius for the various OSG films. The inset presents the same data in the form of $S / 2 a$ versus $a / t$. 


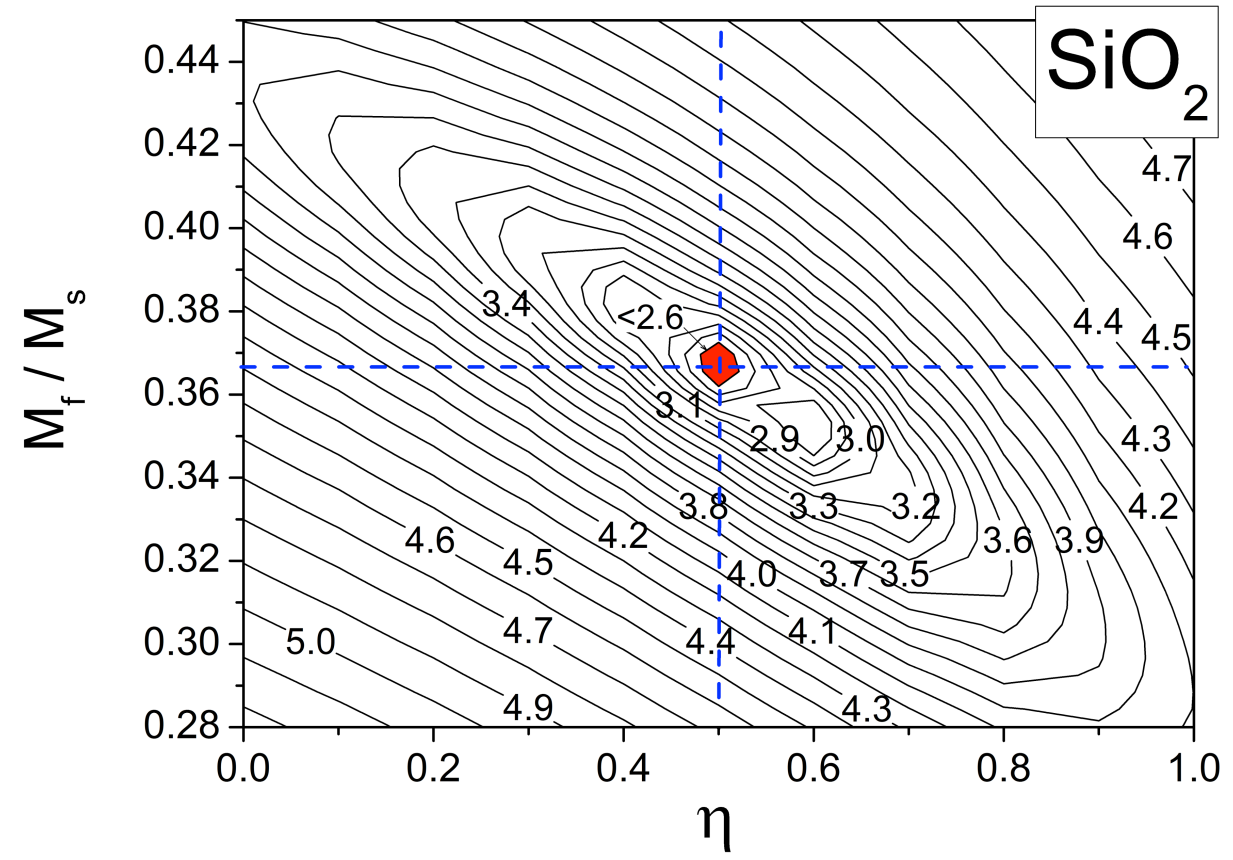

Fig. 12. Contour plot of $\log _{10}\left(\chi^{2}\right)$ as a function of $M_{f} / M_{s}$ and $\eta$ for the $\mathrm{SiO}_{2} / \mathrm{Si}$ sample, with minimum falling within the highlighted region. The unit of $\chi^{2}$ is in $n m^{2}$. 


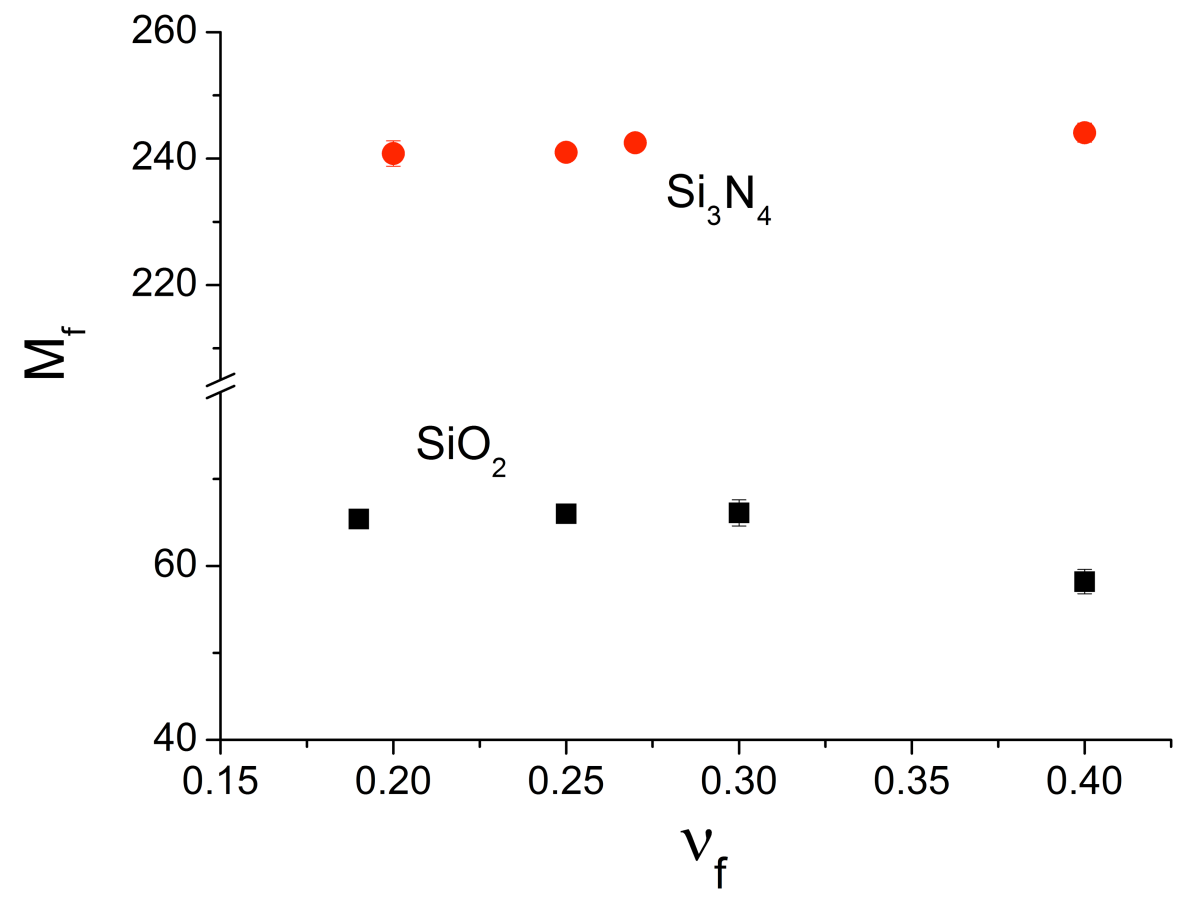

Fig. 13. Indentation moduli of the $\mathrm{SiO}_{2}$ and $\mathrm{Si}_{3} \mathrm{~N}_{4}$ films as a function of the value of Poisson's ratio assumed in the data analysis. 


\section{Tables}

\begin{tabular}{|c|c|c|}
\hline \multirow{2}{*}{$\begin{array}{c}\text { Compliant } \\
\text { film }\end{array}$} & Materials & Thickness (nm) \\
\hline Stiff film & Thermally grown $\mathrm{SiO}_{2}$ & $300 \pm 10$ \\
\hline & OSG-1 & $90 \pm 2$ \\
\cline { 2 - 3 } $\begin{array}{c}\text { Porous } \\
\text { OSG films }\end{array}$ & OSG-2 & $280 \pm 5$ \\
\cline { 2 - 3 } & & $2300 \pm 20$ \\
\cline { 2 - 3 } & & $280 \pm 5$ \\
\hline
\end{tabular}

Table 1. Summary of materials for films and substrate investigated. 


\begin{tabular}{|l|l|l|c|c|}
\hline \multicolumn{1}{|c|}{ Materials } & Poisson's ratio & $\boldsymbol{M ( G P a )}$ & $\boldsymbol{\eta}$ & $\boldsymbol{R}^{\mathbf{2}}$ \\
\hline$(100)-\mathrm{Si}$ & $0.22[12]$ & $178.6 \pm 1.7$ & - & - \\
\hline $\mathrm{SiO}_{2}$ film & $0.19[22 \sim 26]$ & $65.4 \pm 0.7$ & 0.50 & 0.9996 \\
\hline $\mathrm{Si}_{3} \mathrm{~N}_{4}$ film & $0.27[21]$ & $242.5 \pm 0.9$ & 0.55 & 0.9998 \\
\hline OSG-1 (thin) & 0.25 & $4.45 \pm 0.19$ & 1.10 & 0.9992 \\
\hline OSG-1 (thick) & 0.25 & $4.50 \pm 0.20$ & - & - \\
\hline OSG-2 (thin) & 0.25 & $7.07 \pm 0.46$ & 1.20 & 0.9995 \\
\hline OSG-2 (thick) & 0.25 & $7.35 \pm 0.42$ & - & - \\
\hline
\end{tabular}

Table 2. Summary of the nanoindentation results for the various thin-film systems. 


\begin{tabular}{|l|l|l|l}
\hline \multicolumn{1}{|c|}{ Film processing } & $\begin{array}{c}\text { Thickness } \\
(\mathrm{nm})\end{array}$ & \multicolumn{1}{|c}{ Method } & \multicolumn{1}{c}{\begin{tabular}{c}
\multicolumn{1}{c}{ Young's modulus } \\
$(\mathrm{GPa})$
\end{tabular}} \\
\hline $\begin{array}{l}\text { Thermally grown below } 1000^{\circ} \mathrm{C} \\
\text { (present work) }\end{array}$ & $300 \pm 10$ & Nanoindentation & $63.1 \pm 0.7$ \\
\hline $\begin{array}{l}\text { Thermally grown at } 875 \sim 1200^{\circ} \mathrm{C} \\
{[22]}\end{array}$ & $200 \sim 2000$ & Bulge test & $65.2^{*}$ \\
\hline Thermally grown at $960^{\circ} \mathrm{C}[23]$ & 80 & $\begin{array}{l}\text { Micro-beam } \\
\text { resonance }\end{array}$ & 67 \\
\hline Thermally grown $[24]$ & 325 & $\begin{array}{l}\text { Electrically activated } \\
\text { membrane }\end{array}$ & $69 \pm 14$ \\
\hline Thermally grown at $1200^{\circ} \mathrm{C}[25]$ & 650 & $\begin{array}{l}\text { Cantilever beam } \\
\text { technique using } \\
\text { X-ray diffraction }\end{array}$ & $51.3 *$ \\
\hline Thermally grown at $1000^{\circ} \mathrm{C}[26]$ & 1000 & $\begin{array}{l}\text { Brillouin light } \\
\text { scattering technique }\end{array}$ & 72 \\
\hline Bulk fused silica [1] & -- & Nanoindentation & 69.3 \\
\hline
\end{tabular}

* Assume $v=0.19$

Table 3. A survey of Young's moduli for thermally grown $\mathrm{SiO}_{2}$ films reported in the literature. 


\section{Appendix: Step-by-step instructions to implement the proposed method}

\section{Steps to get the experimental $a \sim S$ relation as function of $M_{f}$ and $\eta$}

1. Assume initial values of $M_{f}$ and $\eta$

2. Calculate the effective thickness $t_{\text {eff }}$ for a given point on the indentation loading curve using Eq. 16.

3. Obtain the experimental value of the contact radius at this loading point by solving the following implicit equation numerically:

$$
a_{\mathrm{exp}}=\sqrt{\frac{1}{\pi} f\left[h-\xi\left(\left.\frac{a_{\mathrm{exp}}}{t_{\text {eff }}}\right|_{h}, \frac{M_{f}}{M_{s}}\right) \varepsilon \frac{P}{S}\right]},
$$

where $f$ is the area function of the indenter tip and $\xi$ is obtained from Yu's solution.

4. Calculate the reduced stiffness to remove the compliance of the indenter tip using

$$
S_{r}=\left(\frac{1}{S}-\frac{1}{S_{t i p}}\right)^{-1}
$$

where $S_{t i p}=2 a_{\exp } M_{t i p}$ and $M_{t i p}=1146.6 \mathrm{GPa}$ for a diamond indenter.

5. Repeat steps 2 through 4 for every point of the indentation loading curve to obtain the experimental $\left[S_{r}, a_{\exp }\right]$ relation for the values of $M_{f}$ and $\eta$ assumed in step 1.

\section{Steps to get the theoretical $\boldsymbol{a} \sim \boldsymbol{S}$ relation as a function of film modulus and $\boldsymbol{\eta}$}

6. Assume the same initial values of $M_{f}$ and $\eta$ as in step 1 .

7. Calculate the effective thickness $t_{\text {eff }}$ for a given point on the indentation loading curve using Eq. 16.

8. Calculate the elastic $S \sim a$ relation directly from Yu's solution by using Eqs. (9) (14).

9. Calculate the theoretical contact area $a^{*}$ where the contact stiffness equals $S_{r}$ (as from step 4), from the elastic $S \sim a$ relation.

10. Repeat step 6 through 9 for every point of the indentation loading curve to obtain the theoretical $\left[S_{r}, a^{*}\right]$ relation for the values of $M_{f}$ and $\eta$ assumed in step 1.

\section{Steps to extract unknown film modulus}

10. Compute the $\chi^{2}$, sum of residues squared, using the following formula:

$$
\chi^{2}=\sum\left(a_{\exp }-a^{*}\right)^{2}
$$

11. Find the values of $M_{\mathrm{f}}$ and $\eta$ that minimize $\chi^{2}$ using a standard optimization 
algorithm. 